\title{
A SUFFICIENT CONDITION FOR GRAPHS TO BE SUPER $k$-RESTRICTED EDGE CONNECTED ${ }^{1}$
}

\author{
SHIYING WANG \\ College of Mathematics and Information Science \\ Engineering Laboratory for Big Data Statistical Analysis and Optimal Control \\ Henan Normal University, Xinxiang, Henan 453007, P.R. China \\ e-mail: wangshiying@htu.edu.cn \\ MEIYU WANG \\ School of Mathematical Sciences \\ Shanxi University, Taiyuan, Shanxi 030006, P.R. China \\ e-mail: wangmeiyu6539@126.com \\ AND \\ LEI ZHANG \\ School of Mathematics \\ Jinzhong University, Jinzhong, Shanxi 030600, P.R. China \\ e-mail: zhanglei84052501@163.com
}

\begin{abstract}
For a subset $S$ of edges in a connected graph $G, S$ is a $k$-restricted edge cut if $G-S$ is disconnected and every component of $G-S$ has at least $k$ vertices. The $k$-restricted edge connectivity of $G$, denoted by $\lambda_{k}(G)$, is defined as the cardinality of a minimum $k$-restricted edge cut. Let $\xi_{k}(G)=$ $\min \{|[X, \bar{X}]|:|X|=k, G[X]$ is connected $\}$, where $\bar{X}=V(G) \backslash X$. A graph $G$ is super $k$-restricted edge connected if every minimum $k$-restricted edge cut of $G$ isolates a component of order exactly $k$. Let $k$ be a positive integer and let $G$ be a graph of order $\nu \geq 2 k$. In this paper, we show that if $|N(u) \cap N(v)| \geq k+1$ for all pairs $u, v$ of nonadjacent vertices and $\xi_{k}(G) \leq$ $\left\lfloor\frac{\nu}{2}\right\rfloor+k$, then $G$ is super $k$-restricted edge connected.
\end{abstract}

Keywords: graph, neighborhood, $k$-restricted edge connectivity, super $k$ restricted edge connected graph.

2010 Mathematics Subject Classification: 05C40.

\footnotetext{
${ }^{1}$ This work is supported by the National Science Foundation of China (61370001).
} 


\section{REFERENCES}

[1] J.A. Bondy and U.S.R. Murty, Graph Theory (New York, Springer, 2008).

[2] C. Balbuena, P. García-Vázquez and X. Marcote, Sufficient conditions for $\lambda^{\prime}$ optimality in graphs with girth $g$, J. Graph Theory 52 (2006) 73-86. doi:10.1002/jgt.20150

[3] N.-W. Chang, C.-Y. Tsai and S.-Y. Hsieh, On 3-extra connectivity and 3-extra edge connectivity of folded hypercubes, IEEE Trans. Comput. 63 (2014) 1594-1600. doi:10.1109/TC.2013.10

[4] A.-H. Esfahanian and S.L. Hakimi, On computing a conditional edge-connectivity of a graph, Inform. Process. Lett. 27 (1988) 195-199. doi:10.1016/0020-0190(88)90025-7

[5] J. Fàbrega and M.A. Fiol, Extraconnectivity of graphs with large girth, Discrete Math. 127 (1994) 163-170. doi:10.1016/0012-365X(92)00475-7

[6] Q. Liu, X. Huang and Z. Zhang, Optimally restricted edge connected elementary Harary graphs, Theoret. Comput. Sci. 497 (2013) 131-138. doi:10.1016/j.tcs.2011.12.015

[7] J. Meng, Optimally super-edge-connected transitive graphs, Discrete Math. 260 (2003) 239-248. doi:10.1016/S0012-365X(02)00675-1

[8] J. Meng and Y. Ji, On a kind of restricted edge connectivity of graphs, Discrete Appl. Math. 117 (2002) 183-193. doi:10.1016/S0166-218X(00)00337-1

[9] L. Shang and H. Zhang, Super restricted edge-connectivity of graphs with diameter 2, Discrete Appl. Math. 161 (2013) 445-451. doi:10.1016/j.dam.2012.08.030

[10] M. Wang and Q. Li, Conditional edge connectivity properties, reliability comparisons and transitivity of graphs, Discrete Math. 258 (2002) 205-214. doi:10.1016/S0012-365X(02)00299-6

[11] S. Wang, L. Zhang and S. Lin, A neighborhood condition for graphs to be maximally k-restricted edge connected, Inform. Process. Lett. 112 (2012) 95-97. doi:10.1016/j.ipl.2011.10.012

[12] S. Wang, J. Li, L. Wu and S. Lin, Neighborhood conditions for graphs to be super restricted edge connected, Networks 56 (2010) 11-19. doi: $10.1002 /$ net.20343

[13] S. Wang and L. Zhang, Sufficient conditions for $k$-restricted edge connected graphs, Theoret. Comput. Sci. 557 (2014) 66-75. doi:10.1016/j.tcs.2014.08.018

[14] S. Wang, S. Lin and C. Li, Sufficient conditions for super $k$-restricted edge connectivity in graphs of diameter 2, Discrete Math. 309 (2009) 908-919. doi:10.1016/j.disc.2008.01.037 
[15] M. Zhang, J. Meng, W. Yang and Y. Tian, Reliability analysis of bijective connection networks in terms of the extra edge-connectivity, Inform. Sci. 279 (2014) 374-382. doi:10.1016/j.ins.2014.03.125

Received 13 August 2015

Revised 11 May 2016

Accepted 11 May 2016 